\title{
Giza Pyramid and Havan Kund: Scientific and Spiritual Correlations
}

\author{
Jai Paul Dudeja \\ Professor and Director, Amity University Haryana, Gurgaon 122413, India. \\ drjpdudeja@gmail.com, Mobile: 91-9312247845
}

\begin{abstract}
The Great Pyramid of Giza in Egypt is the oldest and the only one out of the seven wonders of the ancient world that has survived till date. It is believed by some people that the ancient Vedic knowledge and concept was implemented for the design of this and similar pyramids. This is mainly based on the principle of the 'Golden Ratio'. The 'inverted-pyramid-shaped' Havan Kund, which is used by tens of millions of people for performing daily yagyas, is also based on the same concept and design as that of the Pyramid of Giza. Whereas the Pyramids like that of Giza possess the spiritual potential energy, which is used for the benefit of human and plant life placed in them; the Havan Kund is known to spread the spiritual kinetic energy for the benefit of humans, plants and the environment. In this paper, the scientific and spiritual correlations of the Giza Pyramid and the Havan Kund are studied in details. Based on the analysis of these correlations, a new design of Havan Kund is proposed herewith, which is humbly believed to be more effective spiritually and scientifically than the existing Havan Kundas used by many.
\end{abstract}

Key words: Giza Pyramid, Havan Kund, Science of Havan Kund, Golden Ratio, Spirituality.

\section{INTRODUCTION}

\subsection{What is a Pyramid?}

In geometry, a pyramid is a polyhedron formed by connecting a polygonal base and a point, called the apex. Each base edge and apex form a triangle, called a lateral face. The pyramids are normally made of stones. The Great Pyramid of Giza (also known as the Pyramid of Khufu or the Pyramid of Cheops) is the oldest and largest of the three pyramids in the Giza complex bordering what is now known as El Giza, Egypt. It is the oldest of the Seven Wonders of the Ancient World, and the only one to remain largely intact.

The Pyramid of Giza is shown in the following figure (Fig. 1):

Fig. 1 (The Great Pyramid of Giza)

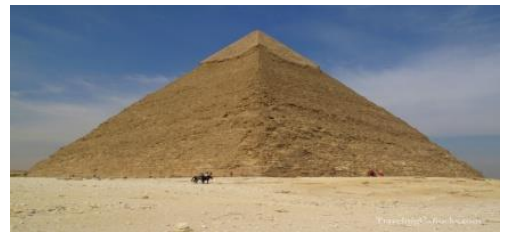

The geometrical outline of the pyramid looks like that depicted in the following figure (Fig. 2):

Fig. 2 (Geometrical outline of pyramid)

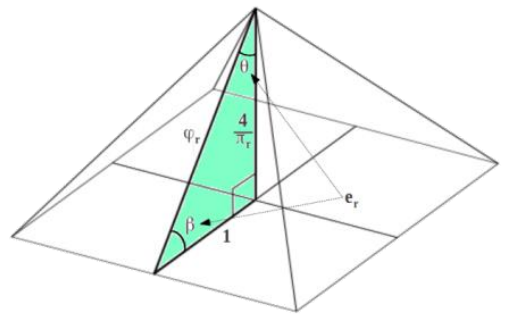

Any one face of the symmetrical pyramid looks like a triangle, as shown in the following figure (Fig. 3):

Fig. 3 (One of the several symmetrical faces of the Giza pyramid)

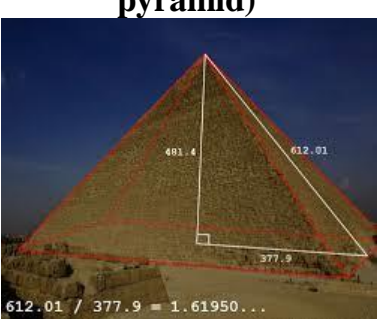

1.2 What is a "Havan Kund"?

A typical 'Havan Kund' is in the shape of an 'inverted pyramid'. It is generally made of metal like, iron or copper. The Havan Kund is used by tens of millions of people across the world, particularly 'Hindus' to sing hymns in praise of the Almighty/Nature/gods (devtas) by performing 'Yagya or Havan'. Agnihotra Havan, for example, can be performed daily by individuals or small groups by offering cow's purified butter (ghee), mixed with wood pieces and many herbal and medicinal items, which are offered to the fire with simultaneous chanting of Vedic mantras. It is believed that the Havan process results to benefit humans, plants, animals and environment. 
International Journal of Advanced Technologies in Engineering and Management Sciences (IJASTEMS-ISSN: 2454-356X) Volume.3,Issue.6,June.2017

The typical geometrical shape of a Havan kund (inverted pyramid), used for Agnihotra, is given in the following figures (Fig. 4, 5):

\section{Fig. 4 (The Havan Kund)}

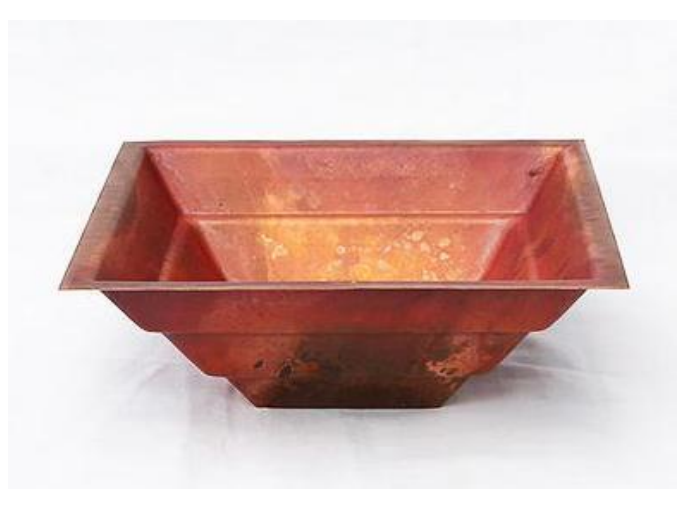

Fig. 5 (Outline of one of the four faces of Havan Kund: the inverted pyramid)

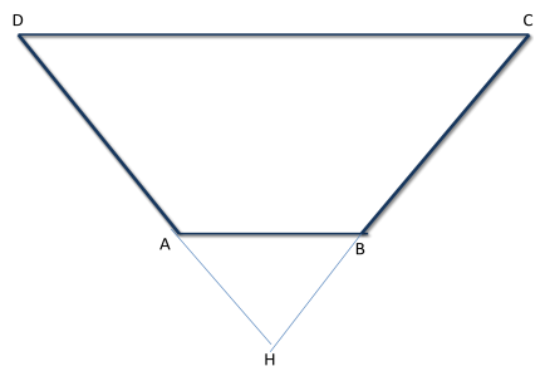

In Fig. 5, ABCD is the boundary of Havan Kund. $A B$ is the length of each side of the lower square and CD is the length of each side of the upper square of the Havan Kund. The lines DA and CB are extended to meet at the point $\mathrm{H}$ to make the geometrical shape look like an 'inverted pyramid', otherwise lines $\mathrm{AH}$ and $\mathrm{BH}$ are not parts of the Havan Kund.

\subsection{A Common Parameter that Connects the} Geometries of Giza Pyramid and Havan Kund:

Whereas the Pyramids like that of Giza possess the spiritual potential energy, which is used for the benefit of human and plant life placed in them; the Havan Kund (the inverted pyramid) is known to spread the spiritual kinetic energy for the benefit of humans, plants and the environment outside the Kund. The common parameter that connects the geometries of Giza Pyramid and Havan Kund is the "Golden Ratio", which is explained below.

\section{THE 'GOLDEN RATIO' AND ITS VEDIC ORIGIN}

\subsection{Introduction to 'Golden Ratio'}

The ratio 1.618 is a golden ratio which is found all over the cosmos [1]. Stick out your forefinger. The ratio from the first two bones is 1.618 . Now the ratio between the 2nd middle bone and the third bone terminating at the knuckle is also 1.618. The ratio from your navel to top of your head and from navel to bottom of your feet is also1.618. This is called the Golden Ratio.

In mathematics, two quantities are in the golden ratio if their ratio is the same as the ratio of their sum to the larger of the two quantities. Expressed algebraically, for quantities $a$ and $b$, with $a>b>0$,

$$
\frac{a+b}{a}=\frac{a}{b}=\varnothing \quad \text { (Golden Ratio) }
$$

For example, if we take $a=1$, then $\frac{1}{b}=\frac{1}{\emptyset}$, and, $1+\frac{1}{\emptyset}=\frac{1}{\emptyset}$

Or

$$
\emptyset^{2}-\emptyset-1=0
$$

which gives

$\emptyset=\frac{1 \pm \sqrt{5}}{2}$

If we take into consideration the - ve sign, it gives $\emptyset_{1}=$ $-0.6180339987$

For the positive sign, it gives

$$
\emptyset_{2}=\varnothing=1.618033988749895 \ldots .(2),
$$

which is the golden ratio.

Further, from Eq. (2), we can see that $\quad \frac{1}{\emptyset}=0.6180339987$.

So that, $\quad \varnothing=1+\frac{1}{\varnothing}=1+\frac{1}{1+\frac{1}{\varnothing}}=1+\frac{1}{1+\frac{1}{1+\frac{1}{\emptyset}}}=$

... and so on .......

\subsection{The Fibonacci Series}

In the twelfth century, an Italian mathematician Leonardo Fibonacci discovered a simple numerical series [2] that is the foundation of an incredible mathematical relationship behind the golden ratio, $\varphi$.

This sequence was known as early as the 6th century AD by Indian mathematicians, but it was Fibonacci who introduced it to the west.

Starting with 0 and 1 , each new number in the sequence is simply the sum of the two before it. $0,1,1,2,3,5,8,13,21,34,55,89,144, \ldots$

The ratio of each successive pair of numbers in the sequence approximates phi $(1.618 .$. ) , as 5 divided by 3 is $1.666 \ldots$, and 8 divided by 5 is 1.60 .

After the 40th number in the sequence, the ratio is accurate to 15 decimal places. $=1.618033988749895$

\subsection{The 'Sri Yantra' and other Vedic Knowledge}

Sri Yantra is the oldest musical instrument, which is believed to be around 10000 years old and is a part of Vedic mathematics [4]. The subsequently known Fibonacci series and the Golden ratio. Sri Yantra is formed by 9 interlocking isosceles triangles. 4 of them 
International Journal of Advanced Technologies in Engineering and Management Sciences (IJASTEMS-ISSN: 2454-356X) Volume.3,Issue.6,June.2017 point upwards and represent the female energy 'Shakti', while the other 5 point downwards, representing the male energy 'Shiva'. These triangles are not ordinarily composed, but have aspects of the Golden Ratio in them. These triangles have 3 variants: the base length, the slant length and the height. The angle also plays a major role. What is amazing is that the triangle of the Yantra is a proportionate cross-section of the Giza Pyramid, incorporating both special numbers pi $(3.142 \ldots)$ and $\varphi$ $(1.618 \ldots)$ ratio. And the base angle of the triangle in the Yantra is seen to be around 51 degrees, the same value that was attributed to the base of the Great Pyramid of Giza. The following figure (Fig. 6) depicts the schematic diagram of 'Sri Yantra'.

Fig. 6 (Sri Yantra)

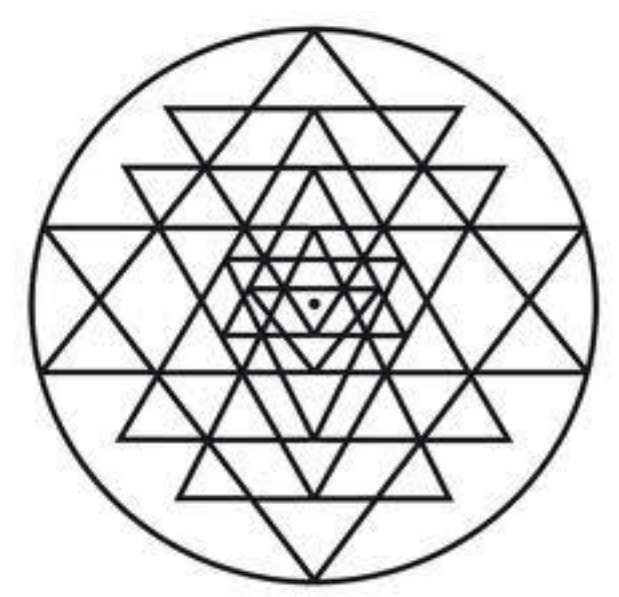

2.4 A Triangle based on the 'Golden Ratio'

Fig. 7 (Triangle based on 'Golden Ratio')

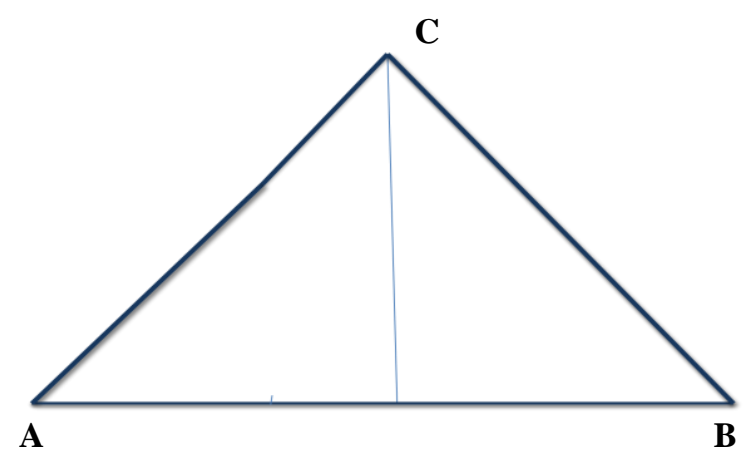

In the above triangle:

$$
\angle \mathrm{CAB}=\angle \mathrm{CBA}=\alpha=51^{0} 49^{\prime} 38.25^{\prime \prime}=51.827^{0}
$$

$$
\angle \mathrm{ACB}=\beta=\left(90^{0}-51.827^{0}\right) \times 2=76.346^{0} .
$$

Therefore, $\angle \mathrm{ACD}=\angle \mathrm{BCD}=\beta / 2=38.173^{\circ}(7)$

If ' $\eta$ ' is any arbitrary real number, we can write

$$
\mathrm{AD}=\mathrm{BD}=\pi \eta=3.14159 \eta,
$$

$\mathrm{AC}=\mathrm{CB}=\pi \varphi \eta($ where $\varphi=$ golden ratio

$$
=1.618034)=5.083199 \eta \text {, }
$$

$$
\text { and } \mathrm{CD}=\pi \eta \sqrt{\varphi}=3.99616 \eta
$$

So we have,

$$
\begin{aligned}
& \sin \alpha=\sin 51.827^{\circ}=0.78615 \\
& \cos \alpha=\cos 51.827^{\circ}=0.61804 \\
& \tan \alpha=\tan 51.827^{0}=1.27201 \\
& \sin \beta / 2=\sin 38.173^{\circ}=0.61804=\cos \alpha \\
& \cos \beta / 2=\cos 38.173^{0}=0.78615=\sin \alpha \\
& \tan \beta / 2=\tan 38.173^{\circ}=0.78616
\end{aligned}
$$

These equations are numbered as these may be used subsequently in this paper.

\section{RETURNING TO THE PYRAMID OF GIZA}

\subsection{Design and Dimensions of the Pyramid of Giza}

The triangle formed by each face of the Pyramid of Giza is exactly identical to Fig. 7.

The actual dimensions of the pyramid are given as following:

$$
\begin{aligned}
& \text { Height }=C D=3.99616 \eta=145.515 \mathrm{~m} \text { (where we have } \\
& \text { used Eq. }(10)) .
\end{aligned}
$$

Base $/ 2=\mathrm{AD}=\mathrm{BD}=3.14159 \eta=115.2815 \mathrm{~m}$ (where we have used Eq. (8)).

Slant Height $=\mathrm{AC}=\mathrm{CB}=5.083199 \eta=186.369 \mathrm{~m}$ (where we have used Eq. (9)).

We can check from Eqns (17-19) that we get $\eta=36.413$, $36.7059,36.6637$, which are of approximately the same values. Therefore each triangle of the Giza pyramid is as per the design given in Fig. 7, which is based on the 'Golden Ratio'.

\subsection{Characteristics of the Pyramid}

Some of the important phenomena observed of the energy concentration within the pyramid are [6]:

(i) Food will not spoil, but will dehydrate, and if kept for a long time, will mummify. 
International Journal of Advanced Technologies in Engineering and Management Sciences (IJASTEMS-ISSN: 2454-356X) Volume.3,Issue.6,June.2017

(ii) Milk does not spoil but becomes cheese over a period of time, without any additives.

(iii) Blades or knives get back their sharp edge if kept for a sufficient number of days.

(iv) Grains germinate quickly, are healthier and grow faster than those planted outside.

(v) Water kept in the pyramid acquires interesting medical properties that help rejuvenate the skin, heal cuts and eliminate many medical problems.

(vi) Larger pyramids where a man can lie down or sit, also help cure certain medical problems and help operations carried out on any part of the body heal faster.

(vii) The use of a pyramid for meditation can accelerate the process of calmness, wellbeing, tranquility, relaxation and freedom from tensions.

\subsection{Standing Waves Generated in the Pyramid}

The base length of the Great Pyramid generates the fundamental resonant tone created by the structures. Each base side of the pyramids has been shown to create a fundamental frequency of about $1.5 \mathrm{~Hz}$ when the pyramids are stimulated into high amplitude [4]. The periodic pulsation of the pyramids operating at resonance creates a Fibonacci standing waves centered on this wavelength bounded by the base of the pyramid. The angles of the standing waves exactly correspond to the slope of the pyramid's faces: in the phi angle of 51.85 degrees. This baseline frequency of $1.5 \mathrm{~Hz}$ has been described as the Tri-thalamic entrainment frequency shown to synchronize the pulsation of the hypothalamus, pineal and pituitary gland into a unified functioning. This frequency is also the lowest frequency of Schumann Resonance, thus the function of the pyramids may indeed be to shift the fundamental frequency of the Earth down from its fluctuations near $7.3 \mathrm{~Hz}$ to the tri-thalamic frequency of $1.5 \mathrm{~Hz}$ [7]. This frequency of $1.5 \mathrm{~Hz}$ falls within the range of delta waves [8]. Delta waves are experienced by the persons in the deep transcendental meditation state. This is the reason why the humans meditating in certain pyramids feel calm, relaxed and energetic.

\section{DESIGN OF HAVAN KUND, THE 'INVERTED PYRAMID'}

\subsection{The Inverted Pyramid Shape}

As depicted in Figs. 4 and 5, the Havan Kund is an inverted pyramid.

\subsection{Geometrical Analysis of the Design of Havan Kund} Based on the correlation from the Giza pyramid, Fig 8 depicts the geometry of the proposed

Havan Kund.

\section{Fig. 8: Geometry of the proposed Havan Kund}

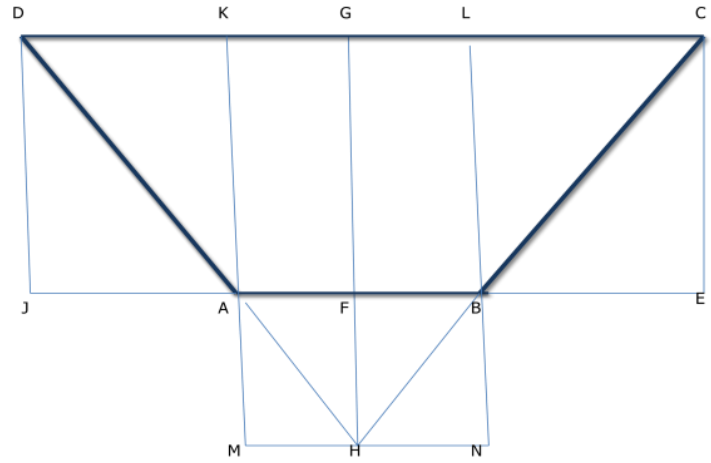

On the lines parallel to discussed in the case of Giza pyramid, we have

$$
\mathrm{DG}=\mathrm{GC}=\mathrm{JF}=\mathrm{FE}==\pi \eta=3.14159 \eta,
$$

$\mathrm{DH}=\mathrm{CH}=\pi \varphi \eta($ where $\varphi=$ golden ratio

$$
\begin{aligned}
=1.618034) & =5.083199 \eta \\
\mathrm{GH} & ==\pi \eta \sqrt{\varphi}=3.99616 \eta
\end{aligned}
$$

Let $A B=x_{1}$ is the length of each side of the lower square of Havan Kund,

$\mathrm{CD}=\mathrm{x}_{2}$ is the length of each side of the upper square of Havan Kund, and

$\mathrm{CE}=$ height of the Havan Kund.

$$
\begin{aligned}
& \angle \mathrm{DAJ}=\angle \mathrm{CBE}=\alpha=51^{0} 49^{\prime} 38.25^{\prime \prime}=51.827^{0} \\
& \angle \mathrm{DHC}=\angle \mathrm{CHD}=\beta=\left(90^{0}-51.827^{0}\right) \times 2 \\
& =76.346^{\circ} .
\end{aligned}
$$$$
\text { Therefore }, \angle D H G=\angle C H G=\beta / 2=38.173^{\circ}
$$

Here $\eta$ can have any arbitrary value, which determines the size and dimensions of the Havan Kund. We can keep changing the value of $\eta$ to determine the size of the Havan kund as per our requirements but the basic geometry remains the same, as explained above.

\subsection{The Proposed New Design of Havan Kund for Better Effects}

As an example, if we decide to fix the base length $\mathrm{AB}$ of the Havan kund equal to $6 \mathrm{~cm}$ and its height equal to 7 $\mathrm{cm}$, we get $\mathrm{CD}=17 \mathrm{~cm}$.

This example of the proposed design of Havan Kund is depicted in Fig. 9, which should give better spiritual and beneficial effects than the ones currently in use [9], which are not exactly following the design of inverted Giza pyramid.

Fig. 9 : Typical dimensions and geometry of the proposed design of Havan Kund 


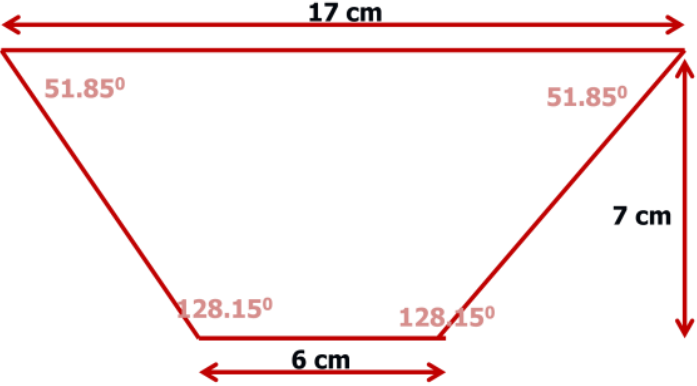

\section{CONCLUSION}

In this paper, we have tried to establish the scientific and spiritual correlations between the oldest surviving pyramid, that is, the Great Pyramid of Giza with the Havan Kund, which is nothing but in the shape of an inverted pyramid. Whereas the Pyramids like that of Giza possess the spiritual potential energy, which is used for the benefit of human and plant life placed in them; the Havan Kund is known to spread the spiritual kinetic energy for the benefit of humans, plants and the environment outside it. It is further shown that these correlations are based on the concept of "Golden Ratio, $\varphi$ " known to the twelfth-century mathematician, Fibonacci or even thousands of years before him as recorded in the Vedic literature. Based on the analysis of these correlations, a new design of Havan Kund is proposed herewith, which is humbly believed to be more effective spiritually and scientifically than the existing Havan Kundas used by many people. It is further suggest that comparative experiments be conducted to study the beneficial effects of Agnihotra Yagya performed by the existing as well as the proposed design of Havan Kundas before arriving at any definitive conclusion.

\section{REFERENCES}

[1] Ramani's blog, "Hinduism Is Mathematics Fibonacci Golden Ratio ....", EducationHealth Hinduism India Lifestyle News Science. https://ramanan50.wordpress.com/2015/03/25/hinduism-ismathematics-fibonacci-golden-ratio-in-meru/

[2] Meisner ,Gary; "Leonardo Fibonacci discovered the sequence which converges on phi”, May 15, 2012.https://www.goldennumber.net/fibonacci-series/

[3] Sanjeev Newar, Sanjeev; "Golden Ratio in Vedas", Agniveer, January 3, 2010. http://agniveer.com/science-in-vedas/
[4] Vadakayil,
Ajit;
"Sri
Yantra,

.......",http://ajitvadakayil.blogspot.in/2010/03/theory-of-

everything-consciousness-and.html/, March 8, 2010.

[5] Meisner ,Gary;"The Great Pyramid of Egypt closely embodies Golden Ratio proportions" . Aug 18, 2012. https://www.goldennumber.net/phi-pi-great-pyramid-egypt/

[6] Poddar, Dr. Prabhat; "The Mysterious Energies Within and Around us",

https://www.sivananda.org/publications/yogalife/fall2003/pdfs/p age49-mysterious-energies.pdf

[7] Putney, Alexander; "Orion Infrasound Pyramid at

Resonance", http://www.human-resonance.org/pyramid.html

[8] Herrmann, Ned; "Brain Frequencies", Scientific American, 22 Dec 1997.

[9] See, for example, Abhang Pranay et al, "Beneficial Effects of Agnihotra on Environment and Agriculture", Int. J. Agri. Sc. And Res., Vol. 5, Issue2, Apr 2015, pp 111-120. 